Special issue of the International Conference on Computational and Experimental Science and Engineering (ICCESEN 2014)

\title{
Calculation of Gamma Strength Functions for Photonucleon Reactions
}

\author{
N. KARPUZ ${ }^{a, *}$, B. MAvi ${ }^{b}$, İ. AKKurT ${ }^{a}$ \\ ${ }^{a}$ Süleyman Demirel University, Physics Department, Isparta, Turkey \\ ${ }^{b}$ Amasya University, Physics Department, Amasya, Turkey
}

\begin{abstract}
The cross section for $(\gamma, \mathrm{N})$ reaction is important for investigation of nuclear structure, especially in low-energy giant dipole resonance (GDR). The total cross sections of ${ }^{12} \mathrm{C}(\gamma, \mathrm{n}){ }^{11} \mathrm{C}$ and ${ }^{12} \mathrm{C}(\gamma, \mathrm{p}){ }^{11} \mathrm{~B}$ reactions, calculated using TALYS 1.2 nuclear code, are 15.5 to $40 \mathrm{MeV}$ and 15 to $110 \mathrm{MeV}$, respectively. In the calculations, the default pre-equilibrium models and Brink-Axel Lorentzian model in all of the gamma strength functions have been used. The effects of the gamma strength function on the cross section exchange data has determined the most compatible model type. The results are compared with the experimental data from the EXFOR database and the evaluated nuclear data from TENDL-2012. Our calculated results are in good agreement with the previously reported experimental results.
\end{abstract}

DOI: 10.12693/APhysPolA.128.B-414

PACS: $24.10 .-\mathrm{i}, 25.20 .-\mathrm{x}$

\section{Introduction}

Nuclear reactions usually involve light particles (p, n, $\alpha, \gamma)$ colliding with a nucleus. Nuclear reactions, however, are usually induced by bombarding a sample with energetic subatomic particles or high-energy photons. The photon is a massless and neutral particle. In comparison with other particles it has a weaker interaction with the target nucleus, and therefore it is less damaging to it. For these reasons it is often preferred over the other particles. At low energies, below about $30 \mathrm{MeV}$, the giant dipole resonance (GDR) is the dominant excitation mechanism, in which collective bulk oscillations of the neutrons with respect to the protons occur [1]. In the giant resonance region, the incident photon interacts with the dipole moment of the target nucleus and the nucleus deexcities by emitting particles or $\gamma$-rays via the compound-nucleus mechanism [2].

Photonuclear reactions are very important for the understanding of the structure of the atomic nucleus and are widely used for variety of applications, such as calculations of the absorbed dose in the human body during radiotherapy, nuclear waste transmutation, astrophysical nucleosynthesis, physics of fusion and fission reactors [3].

\section{Theoretical method and results}

The cross section $(\sigma)$ of a nuclear interaction is a measure of the probability of occurrence of such interaction, which is highly dependent on the energy of the incident particle. In this study, the theoretical photoneutron and photoproton cross sections of ${ }^{12} \mathrm{C}$ in photon-induced reactions have been calculated using TALYS 1.2 nuclear

*corresponding author; e-mail: NurdanKarpuz@hotmail.com calculation code [4]. If the gamma-ray energy is varied by changing the energy of the captured particle, the photonuclear cross section can be determined as a function of photon energy [5]. The calculations on the excitation functions of ${ }^{12} \mathrm{C}(\gamma, \mathrm{n}){ }^{11} \mathrm{C}$ and ${ }^{12} \mathrm{C}(\gamma, \mathrm{p}){ }^{11} \mathrm{~B}$ reactions have been carried out in the incident-photon energy interval between $15.5-40 \mathrm{MeV}$ and $15-110 \mathrm{MeV}$, respectively. The calculated results have been compared with experimental data existing in the EXFOR [6] and TALYS-based Evaluated Nuclear Data Library (TENDL) [7] databases.

TALYS is a nuclear reaction simulation computer code system for the analysis and prediction of nuclear reactions. The basic objective behind its construction is the simulation of nuclear reactions that involve neutrons, photons, protons, deuterons, tritons, $3 \mathrm{He}$ and alpha-particles. TALYS integrates the optical model, direct, preequilibrium, fission and statistical nuclear reaction models in one calculation scheme and gives a prediction for all open reaction channels. There are five models (Kopecky-Uhl generalized Lorentzian, BrinkAxel Lorentzian, Hartree-Fock BCS tables, HartreeFock-Bogolyubov tables, Goriely's hybrid model) for E1 gamma-ray strength function in TALYS. Theoretical cross section calculations are based on these gamma-ray strength function models. Current study was performed for energy range of the giant resonance region (GDR). The total cross section curves obtained for photoneutron and photoproton production in carbon are shown in Figs. 1-10. The contribution of gamma strength functions to photoproton and photoneutron cross-sections in ${ }^{12} \mathrm{C}$ was also considered.

\section{Conclusions}

It can be concluded from this work, that the reaction cross-sections of $(\gamma, \mathrm{n})$ and $(\gamma, \mathrm{p})$, calculated using TALYS 1.2, nuclear calculations code, are in good agreement with the experimental data. The agreement is 


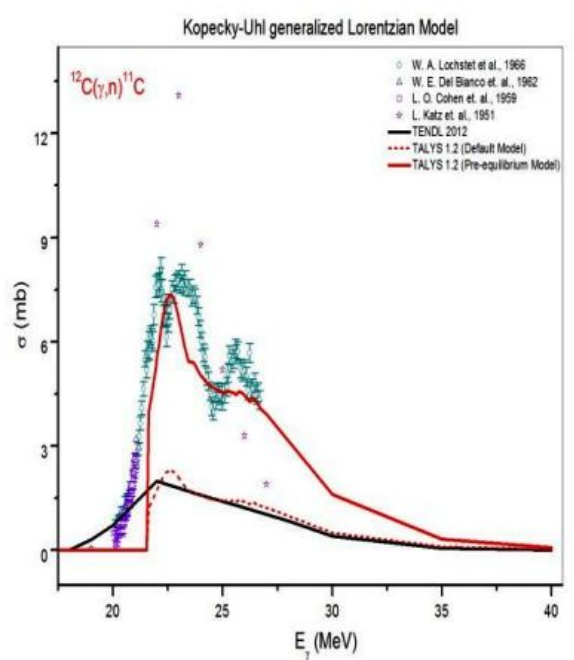

Fig. 1. ${ }^{12} \mathrm{C}(\gamma, \mathrm{n})$ reaction using Kopecky-Uhl generalized Lorentzian model.

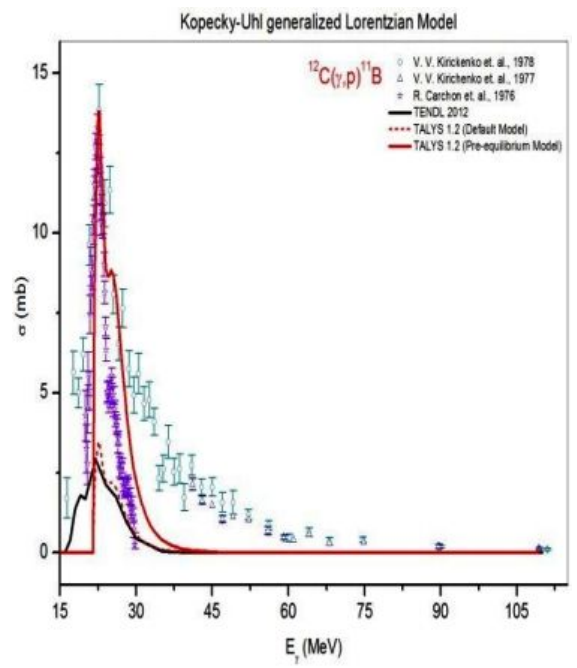

Fig. 2. ${ }^{12} \mathrm{C}(\gamma, \mathrm{p})$ reaction using Kopecky-Uhl generalized Lorentzian model.

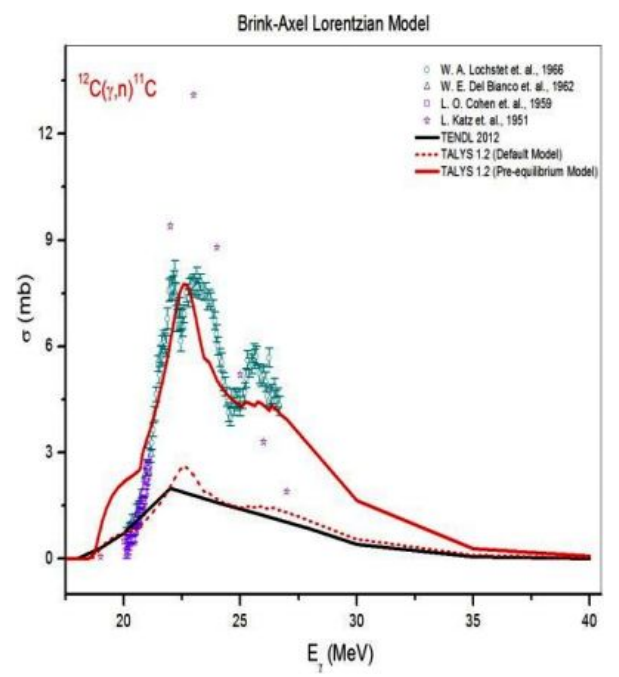

Fig. 3. ${ }^{12} \mathrm{C}(\gamma, \mathrm{n})$ reaction using Brink-Axel Lorentzian model.

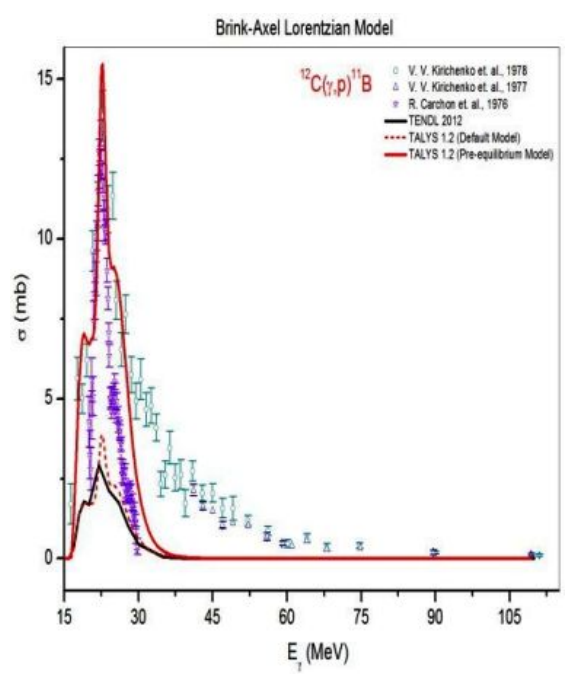

Fig. 4. ${ }^{12} \mathrm{C}(\gamma, \mathrm{p})$ reaction using Brink-Axel Lorentzian model.

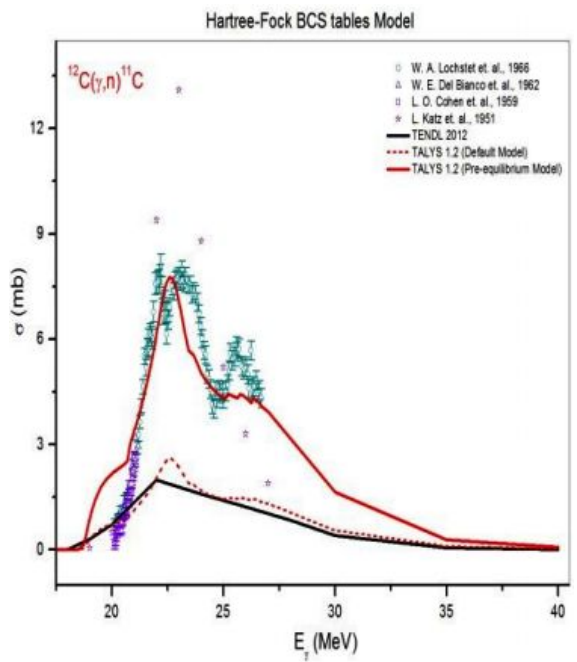

Fig. 5. ${ }^{12} \mathrm{C}(\gamma, \mathrm{n})$ reaction using Hartree-Fock BCS model.

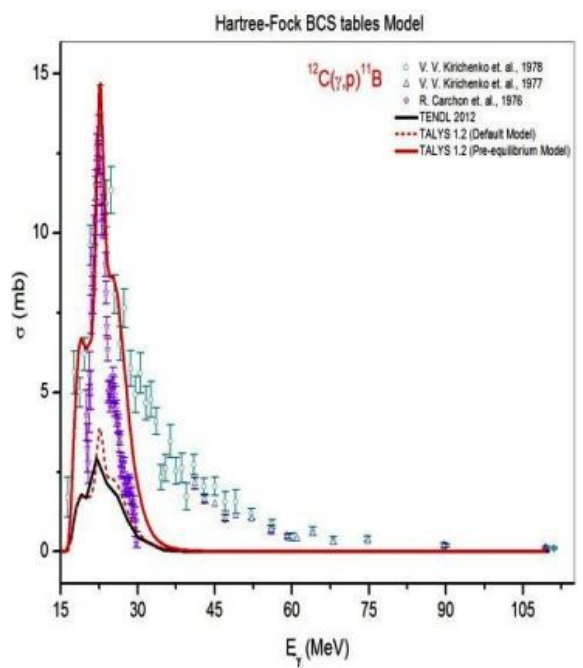

Fig. 6. ${ }^{12} \mathrm{C}(\gamma, \mathrm{p})$ reaction using Hartree-Fock BCS model. 


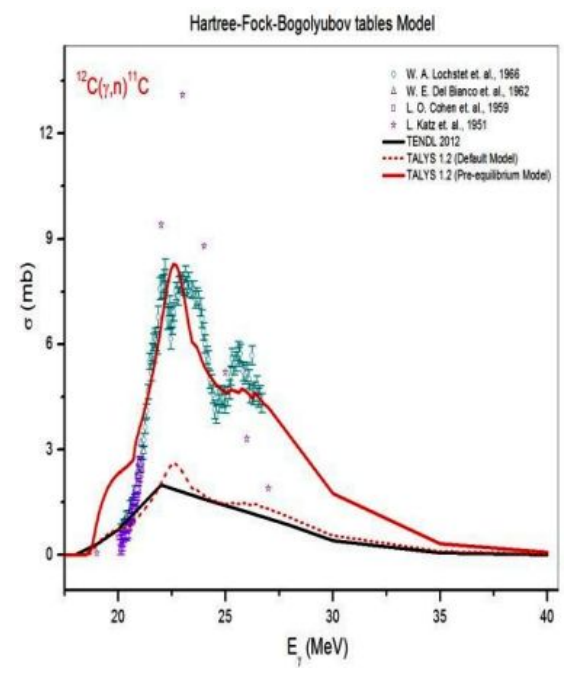

Fig. 7. ${ }^{12} \mathrm{C}(\gamma, \mathrm{n})$ reaction using Hartree-FockBogolyubov model.

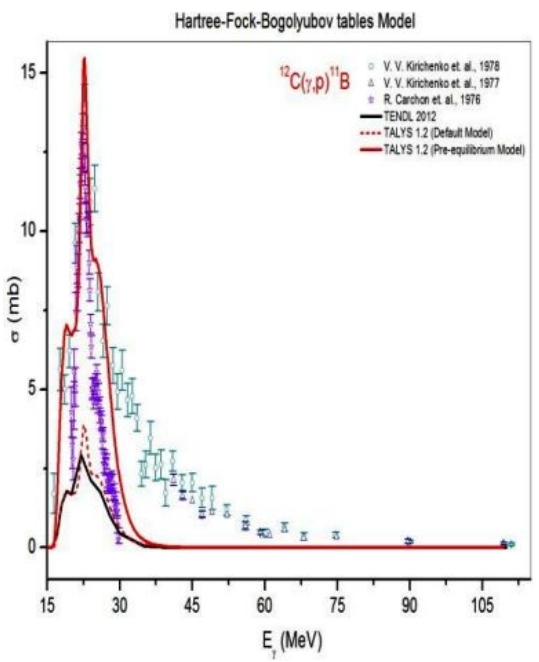

Fig. 8. ${ }^{12} \mathrm{C}(\gamma, \mathrm{p})$ reaction using Hartree-FockBogolyubov model.

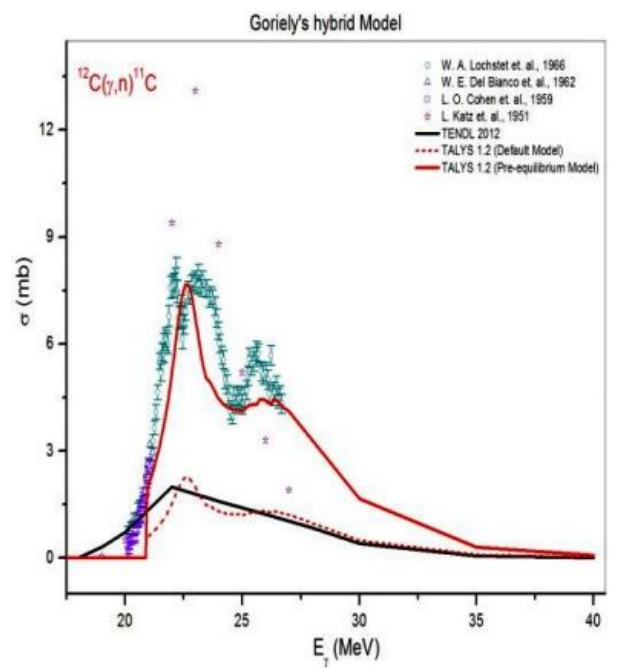

Fig. 9. ${ }^{12} \mathrm{C}(\gamma, \mathrm{n})$ reaction using Goriely's hybrid model.

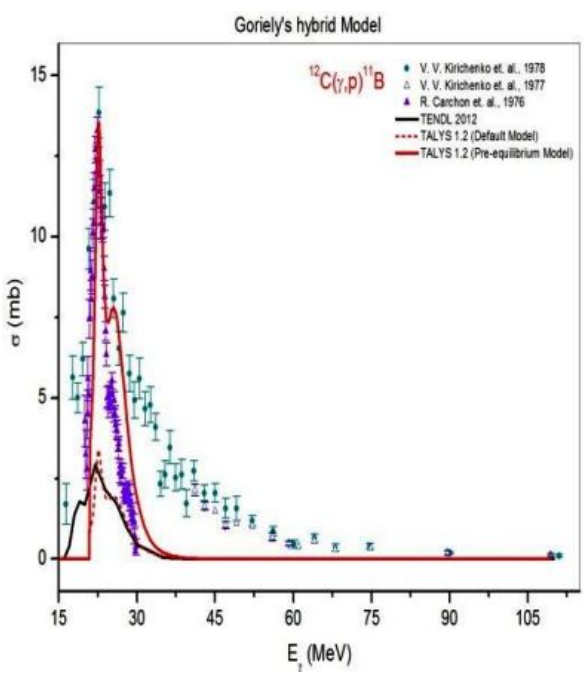

Fig. 10. ${ }^{12} \mathrm{C}(\gamma, \mathrm{p})$ reaction using Goriely's hybrid model.

particularly good in the case of calculated $(\gamma, \mathrm{p})$ reaction cross-sections. The results of theoretical calculations also clearly show that the most appropriate model is the Brink-Axel Lorentzian model.

\section{References}

[1] Y.O. Lee, Y. Han, J.Y. Lee, J. Chanhg, J. Korean Nucl. Soc. 31, 529 (1999).

[2] J.R. Wu, C.C. Chang, Phys. Rev. C 16, (1977).

[3] N. Karpuz, İ. Akkurt, B. Mavi, Ann. Nucl. Energy 60, 341 (2013).

[4] A.J. Koning, S. Hilaire, M. Duijvestijn, TALYS1.2. A nuclear reaction program, User manual, NRG, The Netherlands 2009.

[5] L.D. Cohen, W.E. Stephens, Phys. Rev. Lett. 2, (1959).

[6] EXFOR/CSISRS, Experimental Nuclear Reaction Data File, Brookhaven National Laboratory, National Nuclear Data Center (2009).

[7] TALYS-based Evaluated Nuclear Data Library, TENDL, 2012. 